\title{
A rapid microtitre plate Folin-Ciocalteu method for the assessment of polyphenols
}

Received 26 April 2012; Accepted 24 August 2012

Abstract: Several methods have been described for the determination of phenolic compounds in animal and plant products using the Folin-Ciocalteu (FC) assay. Most of these methods describe the use of this reagent and sodium carbonate in spectrophotometric methods. The macro FC assay was compared with two micro FC assays carried out on a microplate reader. Excellent correlation was obtained among the three assays with a molar extinction coefficient of $5.228 \pm 0.187 \times 10^{3} \mathrm{M}^{-1} \mathrm{~cm}^{-1}$. The micro assay may serve as a high throughput method for the rapid determination of polyphenols in various samples.

Keywords: UV-VIS spectrophotometry • Microplate reader • Methanolic extracts • Tannic acid

(C) Versita Sp. z 0.0 .

\section{Introduction}

Phenolics represent a large number of secondary metabolites present in most fruits and vegetables. These are subsequently divided into the simple phenols or phenolic acids, with one phenol ring and polyphenols, with at least two phenol rings. Typical examples include resorcinol, phloroglucinol, some coumarins, stilbenes, flavonoids, lignans and tannins amongst others.

Several methodshavebeen usedforthe determination of polyphenols in plants. Typically, high performance liquid chromatography $[1,2]$ and spectrophotometric analysis are frequently quoted. More specifically the Folin-Ciocalteu method has been described by many authors [3]. Originally this method was developed for the colorimetric determination of tyrosine, a phenolic nonessential amino acid [4]. Thereafter, this method has been used for the determination of several compounds including plant phenolics, drugs [5-7], vitamin $C$ [8] and other constituents in a wide range of samples ranging from plant extracts to urine and bee products, in particular. The spectrophotometer-cuvette method is also frequently quoted. Little reference is made to the microanalysis using the FC reagent. Although a micromethod has been described for wine phenolics, plastic or glass cuvettes were used [9]. However, this method employs micro volumes for the FC reagent and sodium carbonate. A microplate method has been described specifically for the determination of total phenolic compounds in urine [10] and in food products [11]. The latter method gives a short reaction time of 3 minutes. In most experiments, either gallic acid or tannic acid was used as a phenolic standard.

The present study attempts to demonstrate a rapid method for the determination of polyphenols in a high throughput assay when compared to classical FC assays used.

\section{Experimental Procedures}

\subsection{Materials}

Tannic acid, methanol, Folin-Ciocalteu reagent and sodium carbonate were purchased from Sigma Chemical Co. (St., Louis, USA). Tannic acid was prepared in five 1 in 2 dilutions, from $960 \mathrm{\mu g} \mathrm{ml}^{-1}$ down to $60 \mathrm{\mu g} \mathrm{ml}^{-1}$. The FC reagent was diluted 1:10 with de-ionised water, while sodium carbonate was prepared as a $1 \mathrm{M}$ solution [12]. 


\subsection{Plant materials}

Nine methanolic samples were obtained from orange (Citrus aurantium) peels, aubergine (Solanum melongena) peels and olive (Olea europaea) leaves. Briefly, $5 \mathrm{~g}$ of plant material were allowed to macerate for $48 \mathrm{~h}$ with $30 \mathrm{ml}$ methanol. The resultant mixtures were filtered and the filtrates were mixed with de-ionised water (1:1). All samples were stored in a refrigerator until further analysis.

\subsection{Total polyphenols determination}

The polyphenolic content of the nine extracts, alongside the tannic acid serial dilutions, were tested with the Folin-Ciocalteu reagent using three different detection methods. The general reaction conditions for the three methods are described below and illustrated in Table 1.

A-Absorbance wavelength and time for the reaction. (a) MacroUV assay. The reaction mixtures were allowed to stand for $15 \mathrm{~min}$ and the total phenolic content was determined by colorimetry at 630 and $765 \mathrm{~nm}$ (MU630 and MU765, respectively) in quartz cuvettes on a UV-VIS spectrophotometer (WPA light wave S2000). In order to avoid time-related reading errors, the 15-min reactions were spaced by $1 \mathrm{~min}$. (b) MacroMTP assay. The reaction mixture was prepared for all the extracts and tannic acid dilutions, as for the MacroUV test. During the incubation time, the mixtures were mixed thoroughly and microvolumes of $190 \mu \mathrm{l}$ were analysed at $630 \mathrm{~nm}$ on a Micro titre plate (MTP) reader (BioTek ELx800, Winooski, VT, USA) at the times stipulated below. (c) MicroMTP assay. $10 \mu \mathrm{l}$ of diluted extracts or tannic acid dilutions were pipetted in triplicate in wells of a MTP. The repeated volumes of FC reagent and sodium carbonate were transferred by means of a multichannel pipettor (Gilson). The mixtures were allowed to incubate at room temperature for the times stipulated below and then analysed at $630 \mathrm{~nm}$ on the MTP reader.

Total phenolic values were expressed in terms of tannic acid equivalents $\left(\mu \mathrm{g} \mathrm{ml}^{-1}\right)$. The absorbance readings were taken every five minutes for a period of 60 minutes. The equation $A=\varepsilon$.c.d was applied for the determination of molar extinction coefficient variation between the three assays.
B - Determination of the variation in volume (path length) and absorbance. This was performed for the macroMTP assay. The mixtures were prepared according to the macroUV proportions and mixed thoroughly during the incubation time. Micro-volumes of 300, 250, 190, 150, 100 and $50 \mu \mathrm{l}$ were transferred in triplicate to wells of a MTP. After 20 min incubation, the samples were analysed at $630 \mathrm{~nm}$ on the MTP reader. The equation $A=\varepsilon . c . d$ was used to determine the variation of path length with absorbance for the same reaction mixture (Table 2). The path length was adjusted according to the dimensions of each microtitre plate well and the assay volume used.

C - Detection limit for the reaction. This was conducted for the tannic acid dilutions employing the macroMTP assay. The dilutions considered for this part of the investigation ranged from 960 to $0.002 \mu \mathrm{g} \mathrm{ml}^{-1}$ as 1 in 2 serial dilutions. Following a reaction time of $20 \mathrm{~min}$, the absorbance was read at $630 \mathrm{~nm}$ on the MTP reader.

$D$ - The tested extracts. The methanolic extracts were tested with the three assays (Table 1). For the macroUV assay, the extracts were tested at 630 and $765 \mathrm{~nm}$ (MU630 and MU765). The macroMTP and microMTP assays were tested at 15 and $20 \mathrm{~min}$ (MM15, MM20, $\mathrm{mM} 15$ and $\mathrm{mM} 20$ respectively). The respective tannic acid calibration curve was used to calculate tannic acid equivalents in the extracts.

\subsection{Statistical evaluation}

Comparative analysis was done using Student's t-test (two-tailed) to compare the FC-tannic acid reactions at different time intervals, different volumes and different experiments, alongside the nine extracts tested at a significance level of $P<0.05$. The molar extinction coefficients for the three assays for the tannic acid dilutions and the tannic acid equivalence of the nine extracts were investigated with multivariate analysis. The correlation matrix was calculated, giving the correlation coefficients between each pair of variables tested. To identify variability and to reduce the dimensions of the data set, principal component analysis (PCA) was performed, using the XLSTAT Version 2011.5.01 software (Addinsoft, USA).

\begin{tabular}{|c|c|c|c|c|c|c|}
\hline Method & Reaction vessel & Instrument & $\begin{array}{l}\text { Reaction volume } \\
\qquad(\mathrm{ml})\end{array}$ & $\begin{array}{c}\text { Folin- } \\
\text { Ciocalteu }\end{array}$ & $\begin{array}{l}\text { Sodium } \\
\text { carbonate }\end{array}$ & $\begin{array}{l}\text { Extract or } \\
\text { standard }\end{array}$ \\
\hline MacroUV & Cuvette & UV-VIS spectro-photometer & 9.500 & $5 \mathrm{ml}$ & $4 \mathrm{ml}$ & $0.5 \mathrm{ml}$ \\
\hline MacroMTP & Micro Titre Plate & Micro Titre Plate reader & 0.190 & \multicolumn{3}{|c|}{$190 \mu \mathrm{l}$ of Macro UV reaction } \\
\hline MicroMTP & Micro Titre Plate & Micro Titre Plate reader & 0.190 & $100 \mu \mathrm{l}$ & $80 \mu \mathrm{l}$ & $10 \mu \mathrm{l}$ \\
\hline
\end{tabular}

Table 1. The general reaction conditions for the three methods. 


\section{Results and Discussion}

\subsection{Absorbance wavelength and reaction time} Absorbance wavelength. As described in previous works, the Folin-Ciocalteu reaction with polyphenols produces maximal absorbance between 725 and $765 \mathrm{~nm}$. In this present study, the main peak was determined to be $765 \mathrm{~nm}$. A UV-VIS spectrophotometer is versatile as it can operate at any wavelength between 200 and $950 \mathrm{~nm}$. Usually the standard MTP readers have five fixed wavelength filters and can be operated at these wavelengths only unless the filters are exchanged for different wavelengths. The versatility of the MTP reader is in its ability to read a large number of samples within a very short time interval, as opposed to the UV-VIS spectrophotometer. A suitable wavelength on the MTP reader close to the reaction maximum peak was $630 \mathrm{~nm}$. The macroUV test was carried out with these two wavelengths $(630 \mathrm{~nm}$ and $765 \mathrm{~nm})$ to determine the correlation between reactions for the tannic acid dilutions for the standard curve and the plant extracts.

The molar extinction coefficient for the reaction at path lengths of $1 \mathrm{~cm}$ for the macroUV and $0.5938 \mathrm{~cm}$ for the macroMTP and microMTP was $5.228 \pm 0.187 \times 10^{3} \mathrm{M}^{-1} \mathrm{~cm}^{-1}$. The extinction coefficients values for each individual test were not significantly different $(P>0.05, v=5)$ from each other. From the Principal Component Analysis for the molar extinction coefficients derived from individual determinations for the three assays (i.e. three different experiments on three dates), the correlation matrix exhibited positive correlations (Table 3). It is likely that the MicroMTP and MacroMTP correlate well as they are carried out on a small volume scale with a distinct pathlength, while the MicroMTP and Macro UV correlate well, as the former is a downsizing of the latter reaction and therefore the stoichiometry of the reaction is the same in both assays.

Reaction time. Although the first 60 minutes of reactions were considered, beyond the 35-min interval the absorbance values remained relatively stable with no further changes in absorbance with time. For each concentration, the absorbance at $630 \mathrm{~nm}$ over the time series indicated that the minimum reaction time in the MTP wells was $20 \mathrm{~min}$ compared to the $15 \mathrm{~min}$ in the macroUV test.

\subsection{Determination of the variation in volume (path length) and absorbance}

When using the MTP methods, the path length is not constant as with the UV method, where the reaction mixture is placed in a cuvette of a fixed path length. In the latter case, $10 \mathrm{~mm}$ quartz cuvettes were used. In the MTP method, the path length varies with the reaction

\begin{tabular}{c|c}
\hline Volume of mixture $(\mu \mathrm{l})$ & Path length $(\mathrm{mm})$ \\
\hline 50 & 1.56 \\
100 & 3.13 \\
190 & 5.94 \\
250 & 7.81 \\
300 & 9.38 \\
\hline
\end{tabular}

Table 2. The path length $(\mathrm{mm})$ with mixture volume.

\begin{tabular}{c|ccc}
\hline Variables & MacroUV & MacroMTP & MicroMTP \\
\hline \hline MacroUV & 1 & 0.373 & 0.859 \\
MacroMTP & 0.373 & 1 & 0.796 \\
MicroMTP & 0.859 & 0.796 & 1 \\
\hline
\end{tabular}

Table 3. Correlation matrix for molar extinction coefficients of the three assays

The values at the level of significance, $\alpha=0.050$ (two-tailed t-test).

volume. MTPs with flat-bottomed wells were used in order to maintain a constant surface area throughout all depths. Mixture depths will vary when using U-shaped or V-shaped well plates. With a fixed surface area, the mixture depth or path length can be determined from the volume, as illustrated in Table 2.

\subsection{Detection limit for the reaction}

The maximum concentration for the macro UV was for $480 \mu \mathrm{g} \mathrm{ml}^{-1} \mathrm{TA}$ as the 960 concentration was beyond $2.5 \mathrm{~A}$, which is the upper detection limit for the spectrophotometer. The lower detection limit was determined for the macroMTP method at $630 \mathrm{~nm}$ and a $20 \mathrm{~min}$ reaction time. The limit was $7.5 \mathrm{\mu g} \mathrm{ml}^{-1}$ with tannic acid serving as the reference phenolic compound. There was a good correlation within the 7.5 to $960 \mu \mathrm{g} \mathrm{ml}^{-1}$ range $\left(\mathrm{R}^{2}=0.9974, \mathrm{v}=7\right)$.

\subsection{The tested extracts}

Table 4 represents the percentage (w/w) polyphenols in the plant materials expressed as tannic acid equivalents. The polyphenolic content of the aubergine extracts was low compared to that reported by Jung and co-workers [13], which was $5.519 \%(w / w)$. The olive leaf extracts contained a polyphenolic content equivalent to that reported by Ferreira and co-workers [14], which was $1.271 \%(w / w)$. The orange peel extracts exhibited midrange polyphenolic contents when compared to previous studies, i.e. $0.284 \%, 0.559 \%$ and $0.670 \%$ [15-17].

From the Principal Component Analysis for the percentage polyphenolic content of plant materials, the 


\begin{tabular}{|c|c|c|c|c|c|c|}
\hline & \multicolumn{2}{|c|}{ macroUV } & \multicolumn{2}{|c|}{ macroMTP } & \multicolumn{2}{|c|}{ microMTP } \\
\hline & MU630 & MU765 & MM15 & MM20 & mM15 & mM20 \\
\hline AU1 & $0.250 \pm 0.039$ & $0.247 \pm 0.036$ & $0.186 \pm 0.002$ & $0.189 \pm 0.005$ & $0.175 \pm 0.007$ & $0.167 \pm 0.008$ \\
\hline AU2 & $0.277 \pm 0.024$ & $0.273 \pm 0.020$ & $0.219 \pm 0.002$ & $0.218 \pm 0.006$ & $0.221 \pm 0.004$ & $0.215 \pm 0.006$ \\
\hline AU3 & $0.277 \pm 0.030$ & $0.274 \pm 0.026$ & $0.225 \pm 0.001$ & $0.224 \pm 0.004$ & $0.224 \pm 0.004$ & $0.218 \pm 0.005$ \\
\hline OR1 & $0.459 \pm 0.028$ & $0.452 \pm 0.025$ & $0.385 \pm 0.004$ & $0.391 \pm 0.003$ & $0.344 \pm 0.001$ & $0.344 \pm 0.007$ \\
\hline OR2 & $0.411 \pm 0.044$ & $0.402 \pm 0.039$ & $0.275 \pm 0.039$ & $0.280 \pm 0.044$ & $0.267 \pm 0.007$ & $0.265 \pm 0.009$ \\
\hline OR3 & $0.494 \pm 0.046$ & $0.481 \pm 0.041$ & $0.379 \pm 0.003$ & $0.385 \pm 0.003$ & $0.365 \pm 0.016$ & $0.365 \pm 0.013$ \\
\hline OLC7 & $1.012 \pm 0.002$ & $0.955 \pm 0.008$ & $0.924 \pm 0.008$ & $0.936 \pm 0.013$ & $0.866 \pm 0.017$ & $0.864 \pm 0.012$ \\
\hline OLN7 & $1.161 \pm 0.001$ & $1.084 \pm 0.009$ & $1.044 \pm 0.004$ & $1.058 \pm 0.017$ & $0.998 \pm 0.013$ & $0.996 \pm 0.017$ \\
\hline OLB7 & $1.348 \pm 0.038$ & $1.256 \pm 0.043$ & $1.235 \pm 0.016$ & $1.250 \pm 0.037$ & $1.176 \pm 0.017$ & $1.168 \pm 0.013$ \\
\hline
\end{tabular}

Table 4. The $\% \mathrm{w} / \mathrm{w}$ polyphenols in the plant materials expressed as tannic acid equivalents.

\begin{tabular}{c|cccccc}
\hline Variables & MU630 & MU765 & MM15 & MM20 & mM15 & mM20 \\
\hline \hline MU630 & 1 & 1.000 & 0.998 & 0.998 & 0.998 & 0.998 \\
MU765 & 1.000 & 1 & 0.998 & 0.998 & 0.997 & 0.998 \\
MM15 & 0.998 & 0.998 & 1 & 1.000 & 1.000 & 1.000 \\
MM20 & 0.998 & 0.998 & 1.000 & 1 & 1.000 & 1.000 \\
mM15 & 0.998 & 0.997 & 1.000 & 1.000 & 1.000 & 1.000 \\
mM20 & 0.998 & 0.998 & 1.000 & 1.000 & 1 \\
\hline
\end{tabular}

Table 5. Correlation Matrix for the $\% \mathrm{w} / \mathrm{w}$ polyphenols in the plant materials expressed as tannic acid equivalents.

Values in bold are different from 0 with a significance level alpha $=0.05$

correlation matrix exhibited strong positive correlations (Table 5). This demonstrates that the extrapolation of plant extract-FC reaction absorbances from the tannic acid calibration curve is consistent for all the conditions mentioned above, mainly at the two wavelengths (630 and $675 \mathrm{~nm}$ ) for the MacroUV assays, and the reaction times of 15 and 20 minutes for the MacroMTP and MicroMTP assays. The score plot reported in Figure 1 indicates that the olive leaf extracts contained a higher concentration of polyphenols than the orange and aubergine extracts. In fact, the main polyphenols of the olive leaf are flavonoids and secoiridoid glycosides $[18,19]$, while orange and aubergine are likely to contain flavonoids and phenolic acids [20,21].

In conclusion, differences between the UV-VIS assay and the MTP assays depend mainly on path lengths and reaction stoichiometry. As illustrated above, there is a practical problem with the macroUV test, due to the limitation of sample handling within a single batch of experiments. With the spacing of extract readings by 1 minute (due to the repeated discarding of the previous solution and adequate rinsing and filling up of the cuvette

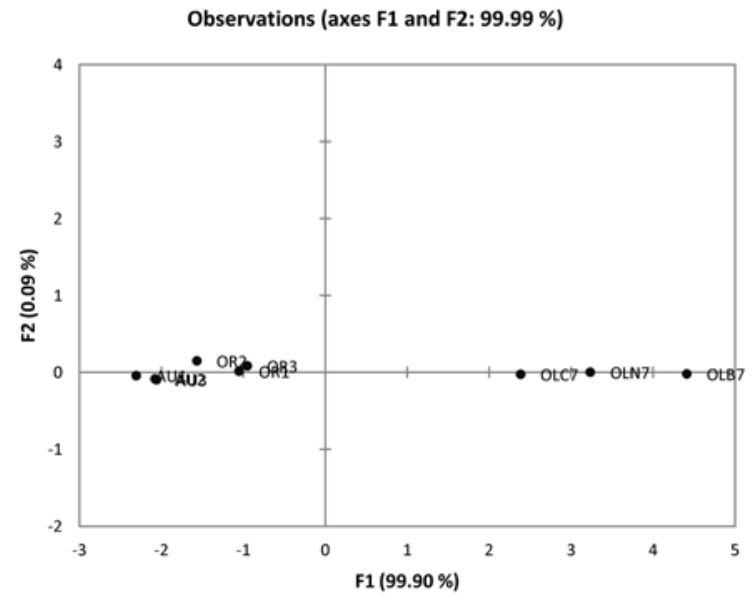

Figure 1. Score plot for the polyphenolic content of olive leaf (OL), orange rind $(\mathrm{OR})$ and aubergine skin (AU) extracts.

with the test solution), only 14 solutions may be tested in a single sequence. Considering that five of these solutions are the standard solutions, only nine extracts can be analysed in such a sequence. To carry out the 
testing in triplicate, the sequence has to be run twice more. The MTP assay offers a faster alternative to this cumbersome method. Testing the solutions in triplicate, with 18 wells dedicated to the tannic acid dilutions and blank, 78 wells are dedicated to 26 extracts in a single sequence. Furthermore, for the amount of reagents required to perform the macroUV and the microMTP with the same number of extracts and replicates, the volumes of FC reagent and sodium carbonate solution would be 50 times more for the macroUV than for the microMTP test, i.e. $480 \mathrm{ml}$ and $384 \mathrm{ml}$, and $9.6 \mathrm{ml}$ and $7.68 \mathrm{ml}$, for the respective tests. The microMTP assay was proven to be better correlated with the macroUV assay as the stoichiometry of the reactants was maintained in both cases. This was observed from the PCA statistical analysis. Furthermore, the optimum reaction time for the microMTP test was 20 minutes, with

\section{References}

[1] Häkkinen S., Heinonen M., Kärenlampi S., Mykkänen H., Ruuskanen J., Törrönen R., Screening of selected flavonoids and phenolic acids in 19 berries, Food Res Int, 1999, 32, 345353

[2] Schieber A., Keller P., Carle R., Determination of phenolic acids and flavonoids of apple and pear by high-performance liquid chromatography, J Chrom., 2001, 910, 265-273

[3] Stratil P., Klejdus B., Kubáň V., Determination of Total Content of Phenolic Compounds and Their Antioxidant Activity in Vegetables - Evaluation of Spectrophotometric Methods, J Agric Food Chem., 2006, 54, 607-616

[4] Folin O., Ciocalteu V., On Tyrosine and Tryptophane Determinations in Proteins, J Biol Chem., 1927, 73, 627-650

[5] Singhvi I., Goyal A., Visible spectrophotometric estimation of aceclofenac and indapamide from tablets using Folin-Cocalteu reagent, Indian J Pharmaceut Sci., 2007, 69, 164-165

[6] Siddappa K., Metre M., Reddy T., Tambe, M., Gavanna M., Sensitive Spectrophotometric Methods for Quantitative Determination of Hydralazine Hydrochloride in Pure and Pharmaceutical Formulation, World J Chem., 2009, 4, 15-20

[7] Singh D.K, Maheshwari G., Spectrophotometric determination of penicillins in pure and pharmaceutical formulations using Folin-Ciocalteu reagent, Drug Test Anal., 2010, 10, 503-506

[8] Jagota S.K., Dani H.M., A new colorimetric technique for estimation of vitamin $\mathrm{C}$ using Folin a detection limit of $7.5 \mu \mathrm{g} \mathrm{ml}^{-1}$. Finally, prior to testing it is advisable that extracts should be scanned within the $600-800 \mathrm{~nm}$ range in order to determine whether other existing compounds could interfere with the chosen FolinCiocalteu absorption wavelengths of 630 or $675 \mathrm{~nm}$. In case extracts interfere at the absorption wavelengths, a MTP with extracts $(10 \mu \mathrm{l})$, de-ionised water $(100 \mu \mathrm{l})$ and sodium carbonate solution $(1 \mathrm{M}, 80 \mu \mathrm{l})$ should be prepared and read at $630 \mathrm{~nm}$ prior to the preparation of the MTP with the reaction mixtures. The absorbance values in the first plate should be subtracted from those of the second plate, to compensate for the background absorbance. Likewise, in case samples contain high amounts of ascorbic acid, especially fruit samples, this oxidation substrate should be determined [22] and subtracted from the total polyphenolic content. This will eliminate possible false positive results.
182

[9] Waterhouse A.L., Determination of total phenolics, In: Wrolstad R.E. (Ed.), Current Protocols in Food Analytical Chemistry, John Wiley \& Sons, New York, 2002

[10] Medina-Remón A., Barrionuevo-González A., Zamora-Ros R., Andres-Lacueva C., Estruch R., Martínez-González M.-A., et al., Rapid FolinCiocalteu method using microtitre 96-well plate cartridges for solid phase extraction to assess urinary total phenolic compounds, as a biomarker of total polyphenols intake, Anal Chim Acta., 2009, 634, 54-60

[11] Magalhães L.M., Santos F., Segundo M.A., Reis S., Lima J.L.F.C., Rapid microplate high-throughput methodology for assessment of Folin-Ciocalteu reducing capacity, Talanta., 2010, 83, 441-447

[12] McDonald S., Prenzler P.D., Autolovich M., Robards K., Phenolic content and antioxidant activity of olive extracts, Food Chem., 2001, 73, 73-84

[13] Jung E.J., Bae M.S., Jo E.K., Jo Y.H., Lee S.C., Antioxidant activity of different parts of eggplant, $\mathrm{J}$ Med Plant Res., 2011, 18, 4610-4615

[14] Ferreira I.C.F.R., Barros L., Soares M.E., Bastos M.L., Antioxidant activity and phenolic contents of Olea europaea L. leaves sprayed with different copper formulations, Food Chem., 2007, 103, 188195

[15] Tzanakis E., Kalogeropoulos Th., Tzimas St., Chatzilazarou A., Katsoyannos E., Phenols and antioxidant activity of apple, quince, pomegranate, 
bitter orange and almond-leaved pear methanolic extracts, E-J Sci Tech., 2006, 1, 16-28

[16] Abd El-aal H.A., Halaweish F.T., Food Preservative Activity of Phenolic Compounds in Orange Peel Extracts (Citrus sinensis L.), Lucrări Ştiinţifice., 2007, 53, 457-464

[17] Magda R.A., Awad A.M., Selim K.A., Evaluation of Mandarin and Navel Orange. Peels as Natural Sources of Antioxidant in Biscuits, Alex J Pharm Sci., 2008, 75-82

[18] Mangion Randon A., Attard E., The in vitro Immunomodulatory Activity of Oleuropein, a Secoiridoid Glycoside from Olea europaea L., Nat Prod Commun., 2007, 2, 515-519

[19] Altıok E., Bayçın D., Bayraktar O., Ülkü S., Isolation of polyphenols from the extracts of olive leaves
(Olea europaea L.) by adsorption on silk fibroin, Sep Purif Technol, 2008, 62, 342-348

[20] Klimczak I., Malecka M., Szlachta M., GliszczynskaSwiglo A., Effect of storage on the content of polyphenols, vitamin $\mathrm{C}$ and the antioxidant activity of orange juices, J Food Comp Anal., 2007, 20, 313-322

[21] Olson R.M., Teo A., Singh A.P., Luthria D., Banuelos G.S., Pasakdee S.M., et al., Polyphenol content and antioxidant capacity of eggplant skin, FASEBJ., 2008, 22, 890.22

[22] Beutler H.-O., L-Ascorbate and L-Dehydroascorbate; In: Bergmeyer H.U. (Ed.), Methods of Enzymatic Analysis (in Methods of Enzymatic Analysis) VCH Publishers (UK) Ltd., Cambridge, UK, 1984 